\title{
Effect of patient education on medication adherence of patients with rheumatoid arthritis: a randomized controlled trial
}

This article was published in the following Dove Medical Press journal: Patient Preference and Adherence

\section{Nichapa Taibanguay \\ Sumapa Chaiamnuay \\ Paijit Asavatanabodee \\ Pongthorn \\ Narongroeknawin}

Division of Rheumatology,

Department of Internal Medicine, Phramongkutklao Hospital and

College of Medicine, Bangkok,

Thailand
Correspondence: Pongthorn Narongroeknawin

Division of Rheumatology, Department of Internal Medicine, Phramongkutklao Hospital and College of Medicine, 315 Ratchawithi Road, Ratchathewi District, Bangkok 10400, Thailand

Tel +6623547980

Fax +662 3544330

Email npongthorn@yahoo.com
Purpose: There is a general understanding that patient educational interventions for enhancing medication adherence are important. However, their success at improving adherence is debatable. This study aimed to assess the influence of different modes of patient education on medication adherence in patients with rheumatoid arthritis (RA).

Materials and methods: One hundred and twenty RA patients with non-adherence, defined as pill count $\leq 80 \%$ or medication-taking behavior questionnaire for Thai patient $\leq 23$, were randomized by block randomization and assigned in a 1:1 allocation ratio to two study arms: multi-component intervention group or single intervention group. The multi-component intervention group received 30-minute directed counseling and a disease information pamphlet. The single intervention group received only a disease information pamphlet. The primary outcomes were an improvement in an adherence rate measured by pill count after 12 weeks. The Thai Clinical Trial Registry number is TCTR20171207003.

Results: After 12 weeks, the pill count adherence rate increased significantly from baseline in both study groups. In the multi-component intervention group, adherence rate increased from $92.21 \pm 14.05$ to $97.59 \pm 10.07(P=0.002)$ and in the single intervention group, it increased from $88.60 \pm 19.66$ to $92.42 \pm 14.27$ ( $P=0.044)$. However, the mean difference between the multicomponent intervention group and the single intervention group was not significant $(5.38 \pm 12.90$ vs $3.18 \pm 14.23, P=0.531$ ). Clinical outcomes, including disease activity score 28 , EuroQoL-5D, EuroQol visual analog scale, pain score, and physician global assessment were unchanged from baseline in both groups.

Conclusion: Patient education significantly improved adherence. However, there were no differences between single education intervention and multi-component education intervention in improving medication adherence. Provision of a disease information pamphlet with or without directed counseling can equally enhance medication adherence of patients with RA.

Keywords: adherence, rheumatoid arthritis, education, disease pamphlet

\section{Introduction}

Rheumatoid arthritis (RA) is a systemic inflammatory condition, affecting from $0.5 \%$ to $1 \%$ of the general population worldwide. The precise causes remain uncertain. A variety of genetic, hormonal, environmental, and infectious agents may contribute to susceptibility and pathogenesis. Inadequately controlled RA leads to serious joint damage, functional disability, morbidity, reduced health-related quality of life, and mortality, which cause a vast economic burden. ${ }^{1,2}$

Prevalence of RA in Thailand is $0.12 \%{ }^{3}$ Chronic rheumatic diseases have a substantial negative effect on both the health and quality of life of patients and their 
caregivers. Moreover, these chronic diseases also have a great influence on society, in terms of health resource utilization, work productivity loss, disability, and death. ${ }^{4}$ In 2007 , the average societal cost of RA in Thailand was $41.1 \%$ of a patient's average annual income, of which, 79.6\% accounted for the direct cost of treatment. ${ }^{5}$

Over the last decade, significant developments have been made in the therapy of RA with the occurrence of novel biological therapies. Several studies have shown that timelier and more aggressive therapy with conventional and biological disease-modifying anti-rheumatic drugs (DMARDs) reduces arthritis symptoms and slows disease progression. However, DMARDs adherence rate in patients with RA is very low, and has varied from $16.4 \%$ to $76.9 \% .^{6-11}$ The nonadherence results in higher disease activity, radiographic damage, disability, a lower quality of life, and a higher health care cost. ${ }^{12-14}$ Multiple factors have been shown to affect medication adherence in patients with RA. However, there are diverging results about the influences of patient and disease characteristics on adherence. ${ }^{7,11,15-33}$

A number of intervention trials have been performed to improve adherence to medication. ${ }^{9,34-42}$ These intervention approaches can be categorized into four groups: educational, behavioral, cognitive behavioral, and multi-component interventions. ${ }^{43}$ Recently, a systematic review determined the impact of different interventions on medication adherence and found that multicomponent interventions had the greatest evidence for improving adherence. ${ }^{36,44}$ However, their effectiveness at enhancing adherence is still uncertain. The National Institute for Health and Care Excellence guideline in 2009 recommended considering any intervention to improve adherence on an individual basis and to modify the intervention to the actual needs of the patients. ${ }^{45}$

This study aimed to assess the impact of different modes of patient education on medication adherence in Thai RA patients.

\section{Materials and methods}

\section{Participants}

Patients who fulfilled the 2010 American College of Rheumatology/European League against Rheumatism criteria for RA were recruited from rheumatology clinic of the Phramongkutklao Hospital from March 2017 to February 2018. Patients were excluded if they were $<18$ years of age, diagnosed with life-threatening conditions, unable to read Thai, unable to take medication by him/herself, had a high disease activity (disease activity score-28, [DAS28] >5.1), or had a severe mental disorder.

\section{Methods}

A total of 185 patients with RA were enrolled. Fifty-six patients with good adherence, defined as medication-taking behavior (MTB) questionnaire for the Thai patient $>23$ or pill count $>80 \%$, were excluded. Seven patients could not follow the study protocol and two denied participating. One hundred and twenty patients with non-adherence were randomized by block randomization and allocated in a 1:1 allocation ratio to two study arms: multi-component intervention group or single intervention group. The multi-component intervention group received a 30-minute directed counseling and a disease information pamphlet. The information pamphlet provided brief information on RA disease and drugs commonly used to treat RA, including usage and dosage information. The single intervention group received only a disease information pamphlet. The study flow chart was depicted in Figure 1.

Baseline demographic and disease characteristics included age, sex, marital status, occupation, salary, year of education, medical insurance, disease duration, tobacco use, alcohol use, comorbidity, rheumatoid factor, anti-citrullinated peptide antibody, dosage and number of DMARDs, and the total number of medicines. All patients were asked to finish a set of standardized self-reporting questionnaires (MTB), patient global assessment (PGA), pain score, EuroQoL-5D (EQ-5D), hospital anxiety and depression scale (HADS), brief illness perception questionnaire (B-IPQ), Montreal cognitive assessment (MoCA), and Thai mental status examination (TMSE). The individual medication was retrieved by querying the electronic medical records combined with patients' self-report. DAS28 and physician global assessment (PhGA) were assessed by blinded rheumatologists. After 12 weeks, patients completed a second set of questionnaires (MTB, PGA, pain score, EQ-5D, HADS, and B-IPQ) and were assessed by the same blinded rheumatologist. One patient in the single intervention group was lost to follow-up.

This study was conducted in accordance with the ethical principles of the Declaration of Helsinki and adhered to the principles outlined in the Guideline for Good Clinical Practice International Conference on Harmonization Tripartite Guideline (January 1997). The study protocol was approved by the institutional review board of the Royal Thai army medical department. Written informed consent was obtained from each patient. The Thai Clinical Trial Registry number is TCTR20171207003.

\section{Pill count adherence rate}

This objective measure counts the number of drugs that have been taken between two visits. This number would then be 


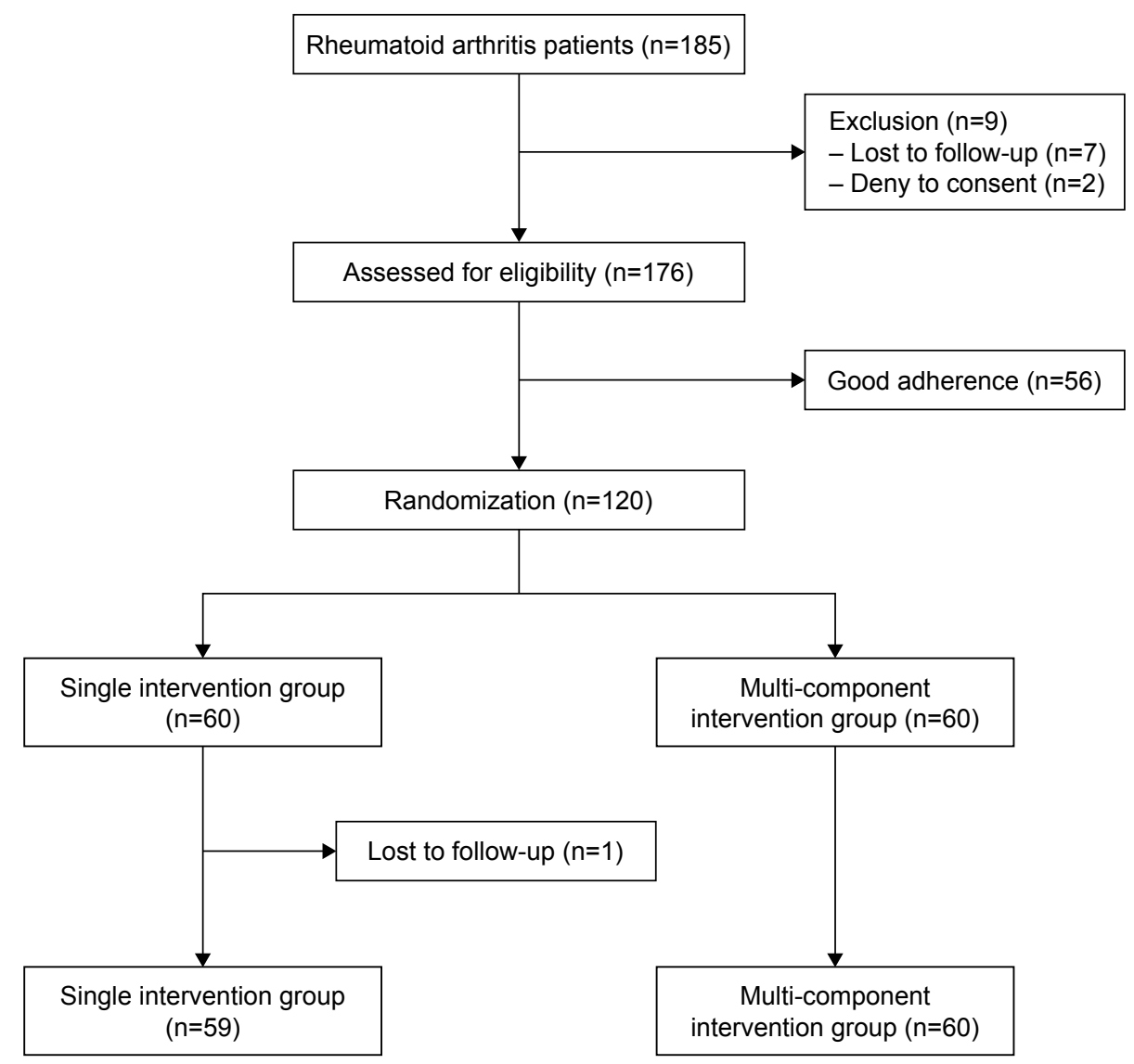

Figure I Patient flow chart.

compared with the total number of drugs received by the patient to calculate the actual adherence rate. ([Number of dosage units dispensed - number of dosage units which remained]/[prescribed number of dosage unit per day $x$ number of days between two visits]). This study categorized adherence as follows: patients with adherence rates $>80 \%$ were considered to be good adherents to the medication, and those with adherence rates $\leq 80 \%$ were considered to be non-adherents to the medication.

\section{Self-reported adherence}

Adherence was also assessed by using the MTB score. This score has been developed and validated in 1,156 Thai patients. It has clearer and more specific language, lower number of items, and better reliability and validity than the Thai version of the 8-item Morisky Medication Adherence Scale (MMAS) to measure medication adherence of the Thai patients. The MTB-Thai consisted of 6 items (forget to take medicines, not taking medicines at times prescribed, stop taking medicines because of adverse drug reactions, stop taking medicines because of getting better, stop taking medicines for other reasons, and adjust dosage regimens) of which, recall periods were 2 weeks. The highest score was 24 . Scores $<22$ represent "low adherence", 22-23 suggest "medium adherence", and equal to 24 indicate "high adherence". ${ }^{46}$

\section{Quality of life}

The general health status of each patient was determined using the EQ-5D-5L. The EQ-5D-5L consists of 2 scales; the EQ-5D-5L descriptive system and the EQ visual analog scale (EQ-VAS). The descriptive system comprises five dimensions and each dimension has five levels. The EQVAS records the patient's health on a $20 \mathrm{~cm}$ vertical VAS. ${ }^{47}$

\section{Disease activity}

Disease activity was assessed by using DAS28, incorporating 28 swollen and tender joint counts, patient's global health assessment on a $100 \mathrm{~mm}$ VAS, and erythrocyte sedimentation rate $(\mathrm{mm} /$ hour $){ }^{48}$

\section{Anxiety and depression}

The HADS was used to measure both anxiety and depression. Each subscale contains seven items, which are rated 
on a 4-point scale and scored from 0 to 3 with total scores varying from 0 to 21 for each subscale. Scores between 8 and 10 suggest a "possible case"; and 11 and 21 designate a "probable case of anxiety/depression". These cutoff points have been found to have sensitivity and specificity of $0.8 .{ }^{49,50}$

\section{Illness perception}

The B-IPQ was utilized to evaluate patients' perceptions of their disease. It comprises 8 items with each level scored from 0 to 10 . Five items measure cognitive illness representations: consequences, identity, personal control, timeline, and treatment control; two items gauge emotional representations: concern and emotional responses; and one item assesses illness comprehensibility. ${ }^{51,52}$

\section{Cognitive assessment}

The Thai version of the MoCA-T test is a translated neuropsychiatric test for measuring the mental status of Thai patients. The MoCA-T measures several cognitive domains, including attention, concentration, executive functions, language, orientation to time and place, short-term memory recall task, visuospatial abilities, and working memory. Education attainment was a significant factor correlated with the score of MoCA-T. The compensation by adding one point for subjects with year of education $\leq 6$ was more appropriate in Thai subjects. The score $\leq 24$ of MoCA-T showed the sensitivity and specificity of $0.8 .^{53}$

The TMSE is another neuropsychiatric test developed for Thais. It consists of six subtests concerning attention, calculation, language, orientation, recall, and registration. The cutoff point for the diagnosis of healthy Thai elderly for TMSE is $>23$ points. This test is sensitive, reliable, and is an applicable mental status examination for Thai subjects who have various socioeconomic status as well as various levels of education and traditional status. ${ }^{54}$

\section{Outcome measures}

The primary endpoints were differences in adherence rates, measured by pill count and MTB after 12 weeks between multi-component intervention group and single intervention group. The secondary endpoints were the changes in RA disease activity as measured by DAS28, pain score, and PhGA by VAS, EQ-5D-5L questionnaire, Thai-HADS questionnaire, and B-IPQ after 12 weeks between multi-component intervention group and single intervention group.

\section{Sample size calculation}

The increase in patient's adherence to treatment was expected to increase from $55 \%$ to $85 \%$. A sample size calculation revealed that a sample of 47 patients in each group was enough to elucidate such difference at 0.05 alpha error and 0.90 power of the test. Sample size calculation was performed using the n4Studies program. ${ }^{55}$

\section{Statistical analyses}

Data were described by standard descriptive statistics. Absolute and relative frequencies were used for categorical variables. Continuous variables were described by mean and range. Randomization was checked by chi-squared test or Fisher's exact test for categorical variables and independent Student's $t$-test for continuous variables. The analysis described was based on an intention-to-treat approach. Significant level was set at $P<0.05$. All statistical analyses were performed with IBM SPSS Statistics for version 23.

\section{Results \\ Demographic measures}

Patient allocation was well balanced. Baseline age, education, income, and underlying disease were not different between the multi-component intervention group and single intervention group (Table 1). The mean age in the multi-component intervention group was $55.82 \pm 11.25$ years, whereas it was $57.20 \pm 12.24$ years in the single intervention group. Females were $83.1 \%$ in the multi-component intervention group, whereas they were $85 \%$ in the single intervention group. There were no differences in disease duration, duration of treatment, extra-articular manifestations, the presence of autoantibodies, disease activity, number of medications, quality of life, illness perception, or cognitive and mental status between the multi-component intervention group and single intervention group. Percentage of patients with anxiety was greater in multi-component intervention group than in single intervention group ( $10 \%$ vs $0 \%, P=0.027)$. The mean disease duration in the multi-component intervention group was $8.29 \pm 7.91$ years, whereas it was $7.73 \pm 6.93$ years in the single intervention group. Baseline pill count adherence rates (92.21 \pm 14.05 vs $88.60 \pm 19.66, P=0.251)$ and MTB-Thai scores $(21.68 \pm 1.91$ vs $21.61 \pm 2.67, P=0.863$ ) were not different (Table 2 ).

\section{Outcome measures}

After 12 weeks, pill count adherence rate increased significantly from baseline in both study groups. In the multicomponent intervention group adherence rate increased from $92.21 \pm 14.05$ to $97.59 \pm 10.07(P=0.002)$ and single intervention group adherence rate increased from $88.60 \pm 19.66$ to $92.42 \pm 14.27$ ( $P=0.044$ ) (Table 3). However, the mean differences between the multi-component intervention group and the single intervention group were not significant (5.38 \pm 12.90 vs $3.18 \pm 14.23, P=0.531$ ) (Table 4). 
Table I Baseline patient characteristics

\begin{tabular}{|c|c|c|}
\hline Variables & $\begin{array}{l}\text { Single intervention } \\
\text { group }(\mathrm{N}=59)\end{array}$ & $\begin{array}{l}\text { Multi-component intervention } \\
\text { group }(\mathrm{N}=60)\end{array}$ \\
\hline Female (\%) & 83.1 & 85 \\
\hline Age (years, mean $\pm S D$ ) & $57.20 \pm 12.24$ & $55.82 \pm 11.25$ \\
\hline $\begin{array}{l}\text { Education attainment ( } \mathrm{n}, \%) \\
\text { Under bachelor's degree } \\
\text { Bachelor's degree or above }\end{array}$ & $\begin{array}{l}69.5 \\
30.5\end{array}$ & $\begin{array}{l}75 \\
25\end{array}$ \\
\hline Years of education (years, mean $\pm S D$ ) & II. $14 \pm 4.32$ & $10.98 \pm 4.12$ \\
\hline $\begin{array}{l}\text { Employment status (n, \%) } \\
\text { Employed } \\
\text { Unemployed }\end{array}$ & $\begin{array}{l}64.4 \\
35.6\end{array}$ & $\begin{array}{l}56.7 \\
43.3\end{array}$ \\
\hline Income ( 10,000 baht $/$ month, mean $\pm S D)$ & $1.25 \pm 1.36$ & $1.49 \pm 1.86$ \\
\hline $\begin{array}{l}\text { Marital status (n, \%) } \\
\text { Single } \\
\text { Married } \\
\text { Divorce } \\
\text { Widow } \\
\end{array}$ & $\begin{array}{l}15.3 \\
59.3 \\
11.9 \\
13.6\end{array}$ & $\begin{array}{l}10 \\
70 \\
5 \\
15\end{array}$ \\
\hline $\begin{array}{l}\text { Social health protection scheme }(\mathrm{n}, \%) \\
\text { CSMBS } \\
\text { Non-CSMBS }\end{array}$ & $\begin{array}{l}49.2 \\
50.8 \\
\end{array}$ & $\begin{array}{l}56.7 \\
43.3\end{array}$ \\
\hline $\begin{array}{l}\text { Underlying disease (n, \%) } \\
\text { Diabetes mellitus } \\
\text { Hypertension } \\
\text { Dyslipidemia } \\
\text { Coronary artery disease } \\
\text { Chronic lung disease } \\
\text { Chronic kidney disease } \\
\text { Osteoporosis }\end{array}$ & $\begin{array}{l}5.1 \\
37.3 \\
32.2 \\
1.7 \\
1.7 \\
0 \\
27.1\end{array}$ & $\begin{array}{l}10 \\
36.7 \\
31.7 \\
5 \\
3.3 \\
1.7 \\
18.3\end{array}$ \\
\hline History of alcohol use (n, \%) & 30.5 & 28.3 \\
\hline History of smoking (n, \%) & 15.3 & 10 \\
\hline Anxiety (n, \%) & 0 & 10 \\
\hline Depression (n, \%) & 1.7 & 6.7 \\
\hline $\begin{array}{l}\text { Cognitive impairment }(\mathrm{n}, \%) \\
\text { TMSE } \\
\text { MoCA-T }\end{array}$ & $\begin{array}{l}11.9 \\
62.7 \\
\end{array}$ & $\begin{array}{l}5 \\
53.3 \\
\end{array}$ \\
\hline $\begin{array}{l}\text { B-IPQ domain (mean } \pm \text { SD) } \\
\text { Consequences } \\
\text { Timeline } \\
\text { Personal control } \\
\text { Treatment control } \\
\text { Identity } \\
\text { Concern } \\
\text { Emotional responses } \\
\text { Illness comprehensibility }\end{array}$ & $\begin{array}{l}4.03 \pm 2.64 \\
5.58 \pm 3.37 \\
5.76 \pm 2.85 \\
8.25 \pm 2.20 \\
3.97 \pm 2.48 \\
4.00 \pm 3.17 \\
7.59 \pm 2.36 \\
4.15 \pm 3.02\end{array}$ & $\begin{array}{l}3.72 \pm 2.74 \\
5.87 \pm 3.62 \\
6.65 \pm 2.90 \\
8.60 \pm 2.25 \\
4.08 \pm 2.66 \\
4.03 \pm 3.32 \\
7.73 \pm 2.82 \\
3.82 \pm 3.24\end{array}$ \\
\hline
\end{tabular}

Abbreviations: B-IPQ, brief illness perception questionnaire; CSMBS, civil servants' medical benefit scheme; HADS, hospital anxiety and depression scale; MoCA-T, Thai version of Montreal cognitive assessment; TMSE, Thai mental state examination.

Sulfasalazine had the lowest adherence rate among other DMARDs. After 12 weeks, adherence rate of sulfasalazine use increased in both groups. In the multi-component intervention group, adherence rate increased from $87.39 \pm 19$ to $97.87 \pm 15.87(P=0.008)$ and in the single intervention group, it increased from $87.94 \pm 17.27$ to $97.74 \pm 8.27$ $(P=0.001)$. However, the mean differences between both groups were not significant $10.48 \pm 23.05$ vs $9.80 \pm 16.64$, $(P=0.887)$ Adherence rate of methotrexate use improved only in the multi-component intervention group 5.76 \pm 16.78 , $(P=0.024)$. The other drugs' adherences were not significantly changed (Table 3 ).

MTB score improved in the multi-component intervention group $(1.12 \pm 2.22, P<0.001)$, but not in the single 
Table 2 Baseline disease and treatment characteristics

\begin{tabular}{|c|c|c|}
\hline Variables & $\begin{array}{l}\text { Single intervention } \\
\text { group }(\mathrm{N}=59)\end{array}$ & $\begin{array}{l}\text { Multi-component intervention } \\
\text { group }(\mathrm{N}=60)\end{array}$ \\
\hline Disease duration (years, mean $\pm S D$ ) & $7.73 \pm 6.93$ & $8.29 \pm 7.91$ \\
\hline Duration of treatment (years, mean \pm SD) & $7.14 \pm 6.59$ & $8.07 \pm 7.94$ \\
\hline $\begin{array}{l}\text { Extra-articular manifestation (\%) } \\
\text { Sicca } \\
\text { Rheumatoid nodule } \\
\text { Interstitial lung disease } \\
\text { Cervical spine involvement }\end{array}$ & $\begin{array}{l}57.6 \\
33.9 \\
28.8 \\
0.0 \\
6.8\end{array}$ & $\begin{array}{l}68.3 \\
46.7 \\
41.7 \\
6.7 \\
3.3\end{array}$ \\
\hline $\begin{array}{l}\text { Autoantibodies } \\
\text { RF positive }(\mathrm{N}=116)(\mathrm{n}, \%) \\
\text { ACPA positive }(\mathrm{N}=92)(\mathrm{n}, \%)\end{array}$ & $\begin{array}{l}71.2 \\
54.2\end{array}$ & $\begin{array}{l}70.0 \\
56.7\end{array}$ \\
\hline Number of medications (mean $\pm S D$ ) & $7.47 \pm 2.18$ & $7.17 \pm 2.34$ \\
\hline $\begin{array}{l}\text { Type and dosage of DMARDs } \\
\text { MTX use }(n, \%) \\
\text { MTX dosage }(\mathrm{mg} / \text { week, mean } \pm \mathrm{SD}) \\
\text { SSZ use }(\mathrm{n}, \%) \\
\text { SSZ dosage }(\mathrm{g} / \text { day, mean } \pm \mathrm{SD}) \\
\text { HCQ use }(\mathrm{n}, \%) \\
\mathrm{HCQ} \text { dosage }(\mathrm{mg} / \text { day, mean } \pm \mathrm{SD}) \\
\text { CQ use }(\mathrm{n}, \%) \\
\text { CQ dosage }(\mathrm{mg} / \text { day, mean } \pm \mathrm{SD}) \\
\text { LEF use }(\mathrm{n}, \%) \\
\text { LEF dosage }(\mathrm{mg} / \text { day, mean } \pm \mathrm{SD}) \\
\text { Prednisolone use }(\mathrm{n}, \%) \\
\text { Prednisolone dosage }(\mathrm{mg} / \text { day, mean } \pm \mathrm{SD}) \\
\text { Biologics use }(\mathrm{n}, \%)\end{array}$ & $\begin{array}{l}76.3 \\
12.17 \pm 3.94 \\
62.7 \\
1.92 \pm 0.76 \\
27.1 \\
179.46 \pm 44.26 \\
16.9 \\
219.70 \pm 65.09 \\
20.3 \\
12.13 \pm 6.44 \\
32.2 \\
5.43 \pm 3.11 \\
1.7\end{array}$ & $\begin{array}{l}81.7 \\
12.14 \pm 4.33 \\
65.0 \\
1.94 \pm 0.70 \\
28.3 \\
179.82 \pm 45.78 \\
8.3 \\
200.00 \pm 68.47 \\
25.0 \\
14.19 \pm 5.73 \\
40.0 \\
4.18 \pm 2.75 \\
1.7\end{array}$ \\
\hline History of adverse drug reaction $(\mathrm{n}, \%)$ & 20.3 & 33.3 \\
\hline DAS28 $($ mean \pm SD) & $3.24 \pm 1.19$ & $3.20 \pm 0.90$ \\
\hline EQ-5D $($ mean $\pm S D)$ & $0.85 \pm 0.12$ & $0.82 \pm 0.17$ \\
\hline EQ-VAS (mean \pm SD) & $79.66 \pm 15.62$ & $77.92 \pm 20.92$ \\
\hline VAS pain $($ mean \pm SD) & $19.14 \pm 20.17$ & $18.33 \pm 21.48$ \\
\hline VAS PhGA mm, (mean \pm SD) & $14.66 \pm 17.29$ & $13.90 \pm 17.03$ \\
\hline $\begin{array}{l}\text { Pill count adherence rate (\%) } \\
\text { Good adherence }(n, \%)\end{array}$ & $\begin{array}{l}88.60 \pm 19.66 \\
16.7\end{array}$ & $\begin{array}{l}92.21 \pm 14.05 \\
27.1\end{array}$ \\
\hline $\begin{array}{l}\text { MTB-Thai 0-24, (mean } \pm \text { SD) } \\
\text { Good adherence }^{\mathrm{b}}(\mathrm{n}, \%)\end{array}$ & $\begin{array}{l}21.61 \pm 2.67 \\
33.3\end{array}$ & $\begin{array}{l}21.68 \pm 1.91 \\
28.8\end{array}$ \\
\hline
\end{tabular}

Notes: ${ }^{a}$ Good adherence defined as pill count adherence rate $>80 \%$. ${ }^{b}$ Good adherence defined as MTB-Thai $\geq 22$.

Abbreviations: ACPA, anti-citrullinated protein antibody; CQ, chloroquine; EQ-5D, EuroQol 5 dimensions; EQ-VAS, EuroQol visual analog scale; DAS28, disease activity score 28; DMARDs, disease-modifying anti-rheumatic drugs; HCQ, hydroxychloroquine; LEF, leflunomide; MTB-Thai, Medication taking behavior questionnaire for Thai patient; MTX, methotrexate; RF, rheumatoid factor; SSZ, sulfasalazine; VAS pain, visual analog scale for pain; VAS PhGA, visual analog scale for physician global assessment.

intervention group $(0.44 \pm 2.92, P=0.0251)$. DAS28, EQ-5D, EQ-VAS, pain, and PhGA were unchanged from baseline in both groups. There was no difference in the proportion of patients who had anxiety or depression. Illness perceptions remained unchanged (Tables 3 and 4).

\section{Discussion}

During a 12-week period, adherence rate measured by pill count in both groups (single intervention and multicomponent intervention) were significantly improved. However, MTB score improved only in multi-component intervention group. Both pill count and MTB score are indirect measurements of adherence. ${ }^{31}$ The result of pill count method is more reliable than MTB score because MTB score is a questionnaire that patients report by themselves. The data suggested that patient education could improve medication adherence. However, there were no differences between single intervention and multi-component intervention groups in improving medication adherence.

A number of intervention trials have been conducted to enhance adherence to treatment in patients with immunemediated inflammatory disorders, including information 
Table 3 Disease parameters at baseline and I 2 weeks of the single and multi-component intervention groups

\begin{tabular}{|c|c|c|c|c|c|c|}
\hline \multirow[t]{2}{*}{ Variables } & \multicolumn{3}{|c|}{ Single intervention group $(\mathrm{N}=59)$} & \multicolumn{3}{|c|}{$\begin{array}{l}\text { Multi-component intervention group } \\
(\mathrm{N}=60)\end{array}$} \\
\hline & At baseline & At I 2 weeks & $P$-value & At baseline & At I 2 weeks & $P$-value \\
\hline $\begin{array}{l}\text { Pill count adherence rate (\%) } \\
\text { Good adherence }^{\mathrm{a}}(\mathrm{n}, \%)\end{array}$ & $\begin{array}{l}88.60 \pm 19.66 \\
72.9\end{array}$ & $\begin{array}{l}92.42 \pm 14.27 \\
89.8\end{array}$ & $\begin{array}{l}0.044 \\
0.019\end{array}$ & $\begin{array}{l}92.21 \pm 14.05 \\
83.3\end{array}$ & $\begin{array}{l}97.59 \pm 10.07 \\
95\end{array}$ & $\begin{array}{l}0.002 \\
0.040\end{array}$ \\
\hline $\begin{array}{l}\text { Adherence rate per each medication } \\
\text { MTX (\%) } \\
\text { SSZ (\%) } \\
\text { HCQ }(\%) \\
\text { CQ (\%) } \\
\text { LEF (\%) } \\
\text { Prednisolone (\%) }\end{array}$ & $\begin{array}{l}89.76 \pm 19.96 \\
87.94 \pm 17.27 \\
88.06 \pm 35.80 \\
95.32 \pm 11.80 \\
87.95 \pm 16.04 \\
94.12 \pm 13.69\end{array}$ & $\begin{array}{l}92.60 \pm 15.01 \\
97.74 \pm 8.27 \\
87.07 \pm 16.00 \\
96.19 \pm 13.50 \\
98.57 \pm 15.00 \\
94.26 \pm 11.91\end{array}$ & $\begin{array}{l}0.190 \\
0.001 \\
0.893 \\
0.788 \\
0.143 \\
0.975\end{array}$ & $\begin{array}{l}92.61 \pm 16.05 \\
87.39 \pm 19.00 \\
89.38 \pm 24.20 \\
105.18 \pm 4.40 \\
100.84 \pm 15.78 \\
90.95 \pm 20.52\end{array}$ & $\begin{array}{l}98.37 \pm 9.70 \\
97.87 \pm 15.87 \\
90.32 \pm 24.87 \\
103.94 \pm 7.53 \\
97.18 \pm 9.31 \\
97.45 \pm 7.00\end{array}$ & $\begin{array}{l}0.024 \\
0.008 \\
0.628 \\
0.585 \\
0.438 \\
0.181\end{array}$ \\
\hline $\begin{array}{l}\text { MTB score }(\text { mean } \pm \text { SD) } \\
\text { Good adherence }^{b}(n, \%)\end{array}$ & $\begin{array}{l}21.61 \pm 2.67 \\
71.2\end{array}$ & $\begin{array}{l}22.05 \pm 1.91 \\
71.2\end{array}$ & $\begin{array}{l}0.251 \\
1.000\end{array}$ & $\begin{array}{l}21.68 \pm 1.91 \\
66.7\end{array}$ & $\begin{array}{l}22.80 \pm 1.34 \\
85\end{array}$ & $\begin{array}{l}<0.001 \\
0.020\end{array}$ \\
\hline DAS28 (mean \pm SD) & $3.24 \pm 1.19$ & $3.17 \pm 1.23$ & 0.563 & $3.20 \pm 0.90$ & $3.01 \pm 1.05$ & 0.160 \\
\hline EQ-5D $($ mean $\pm S D)$ & $0.850 \pm 0.123$ & $0.825 \pm 0.136$ & 0.142 & $0.823 \pm 0.175$ & $0.824 \pm 0.177$ & 0.945 \\
\hline EQ-VAS (mean \pm SD) & $79.66 \pm 15.62$ & $74.64 \pm 20.51$ & 0.055 & $77.92 \pm 20.92$ & $76.08 \pm 21.35$ & 0.577 \\
\hline VAS pain $($ mean \pm SD) & $19.41 \pm 20.17$ & $20.00 \pm 23.27$ & 0.840 & $|8.33 \pm 2| .48$ & $12.58 \pm 17.72$ & 0.092 \\
\hline VAS PhGA (mean $\pm S D)$ & $14.66 \pm 17.29$ & $17.29 \pm 20.75$ & 0.272 & $13.90 \pm 17.03$ & $10.12 \pm 15.62$ & 0.159 \\
\hline $\operatorname{Anxiety}^{c}(\mathrm{n}, \%)$ & 0 & 1.7 & 0.317 & 10 & 3.3 & 0.142 \\
\hline Depression $^{c}(\mathrm{n}, \%)$ & 1.7 & 3.4 & 0.560 & 6.7 & 3.3 & 0.724 \\
\hline $\begin{array}{l}\text { B-IPQ domain (mean } \pm \text { SD) } \\
\text { Consequences } \\
\text { Timeline } \\
\text { Personal control } \\
\text { Treatment control } \\
\text { Identity } \\
\text { Concern } \\
\text { Emotional responses } \\
\text { Illness comprehensibility }\end{array}$ & $\begin{array}{l}4.03 \pm 2.64 \\
5.58 \pm 3.37 \\
5.76 \pm 2.85 \\
8.25 \pm 2.02 \\
3.94 \pm 2.48 \\
4.00 \pm 3.17 \\
7.59 \pm 2.36 \\
4.15 \pm 3.02\end{array}$ & $\begin{array}{l}3.47 \pm 2.57 \\
5.47 \pm 3.37 \\
6.07 \pm 2.77 \\
7.83 \pm 2.08 \\
3.80 \pm 2.34 \\
4.19 \pm 2.84 \\
7.78 \pm 1.90 \\
4.44 \pm 3.05\end{array}$ & $\begin{array}{l}0.160 \\
0.842 \\
0.501 \\
0.173 \\
0.621 \\
0.587 \\
0.551 \\
0.520\end{array}$ & $\begin{array}{l}3.73 \pm 2.77 \\
5.92 \pm 3.63 \\
6.66 \pm 2.93 \\
8.64 \pm 2.24 \\
4.12 \pm 2.67 \\
4.07 \pm 3.34 \\
7.75 \pm 3.84 \\
3.81 \pm 3.27\end{array}$ & $\begin{array}{l}3.54 \pm 2.98 \\
6.32 \pm 3.47 \\
6.19 \pm 3.12 \\
8.54 \pm 2.03 \\
3.95 \pm 2.63 \\
4.08 \pm 3.13 \\
7.95 \pm 2.47 \\
3.49 \pm 2.94\end{array}$ & $\begin{array}{l}0.594 \\
0.423 \\
0.268 \\
0.778 \\
0.621 \\
0.972 \\
0.670 \\
0.329\end{array}$ \\
\hline
\end{tabular}

Notes: ${ }^{\mathrm{a} G o o d}$ adherence defined as pill count adherence rate $>80 \%$. ${ }^{\mathrm{b}} \mathrm{Good}$ adherence defined as MTB-Thai $\geq 22$. 'Anxiety and depression defined as HADS score $>10$. Abbreviations: B-IPQ, brief illness perception questionnaire; CQ, chloroquine; DAS28, disease activity score 28; EQ-5D, EuroQol 5 dimensions; EQ-VAS, EuroQol visual analog scale; HADS, hospital anxiety and depression scale; HCQ, hydroxychloroquine; LEF, leflunomide; MTB-Thai, medication taking behavior questionnaire for Thai patient; MTX, methotrexate; SSZ, sulfasalazine; VAS pain, visual analog scale for pain; VAS PhGA, visual analog scale for physician global assessment.

about disease, ${ }^{41}$ medication reminders using pillbox or mobile phone, ${ }^{56}$ and motivational interview. ${ }^{57}$ A recent systemic analysis revealed that multi-component interventions showed the greatest evidence for promoting adherence in patients. ${ }^{36}$ However, this systematic analysis found a high level of heterogeneity in study methods as well as little consistency in their conclusions, ${ }^{9,40,58,59}$ which does not allow us to draw clear assumptions about the interventions intended to improve medication adherence.

A British randomized controlled study of RA patients revealed that patient education was correlated with adherence.$^{41}$ One hundred active RA patients were randomized to an intervention group, which received seven 30-minute one-on-one sessions with a rheumatology nurse directed at improving self-efficacy, or a control group, which received standard treatment (providing a drug information pamphlet). After 6 months, those in the intervention group were more adherent to drugs. In contrast, a less intensive intervention involving two pharmacist-led motivational interviewing group sessions failed to improve DMARDs adherence more than providing information regarding their medications. ${ }^{60}$

Our study showed no differences between single intervention and multi-component interventions in improving medication adherence. This implied that educational interventions, which deliver instructive information alone, may lead to improved medication adherence. However, the 30-minute directed counseling with provision of brochures did not provide any add-on benefit. One explanation for the imperfect effect of these educational interventions may be that provision of information presumes the patient lacks understanding of their disease and medications. This attributes a passive role to the patient and fails to reflect pre-existing "lay beliefs" acquired from other sources, for example, the newspapers, social media, or friends and family. ${ }^{18}$ A successful educa- 
Table 4 Mean changes in disease parameters from baseline until the 12-week follow-up in both single and multi-component intervention groups

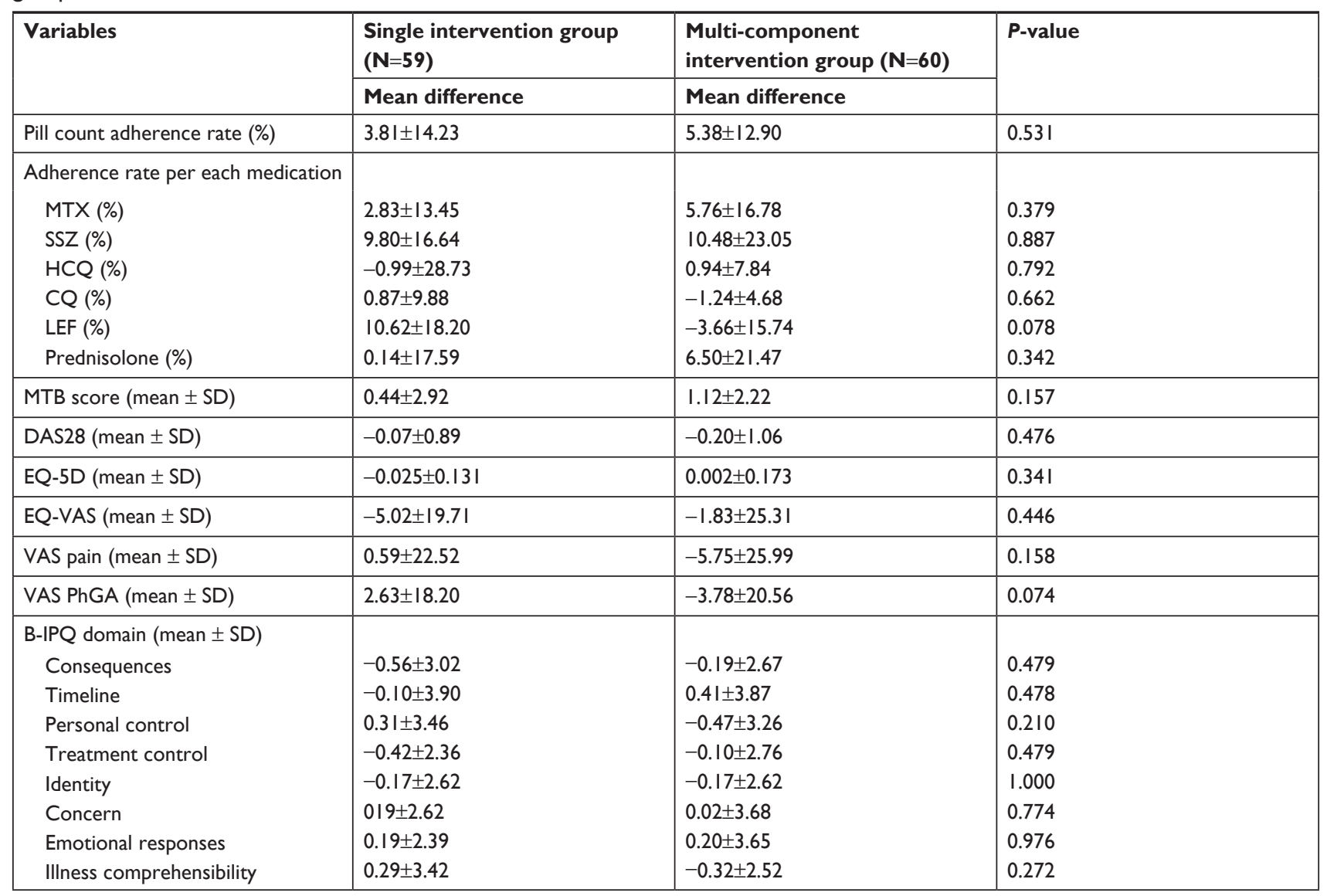

Abbreviations: B-IPQ, brief illness perception questionnaire; CQ, chloroquine; DAS28, disease activity score 28; EQ-5D, EuroQol 5 dimensions; EQ-VAS, EuroQol visual analog scale; HCQ, hydroxychloroquine; LEF, leflunomide; MTB-Thai, medication taking behavior questionnaire for Thai patient; MTX, methotrexate; SSZ, sulfasalazine; VAS pain, visual analog scale for pain; VAS PhGA, visual analog scale for physician global assessment.

tional intervention may require participation from both the physician and patient. Admittedly, some patients may wish to entrust most of the decision making to their treating physician. It is, therefore, crucial to determine how involved the patient wishes to be in constructing the management plan. ${ }^{61}$ As soon as this is established, a proper educational intervention could involve intertwining biomedical information into pre-existing lay belief systems..$^{18}$

Although education interventions in this study improved adherence to DMARD treatment, as in other studies, this boosted adherence did not produce considerable additional beneficial clinical outcome..$^{37,62,63}$ Possibly, the composite index for measuring disease activity and quality of life might not have been a sensitive indicator to detect trivial changes between the two groups.

\section{Limitations}

This study had several limitations. First, patient's adherence is challenging to measure and all techniques have downsides. Both self-reported questionnaire and pill count methods tend to overestimate adherence behavior and generally have low sensitivity. ${ }^{64,65}$ Second, a 12-week study period may perhaps be too short to affect the clinical outcome. A longer time period might be needed to demonstrate maximal efficacy. Third, nearly one-fourth of patients in this trial reported a perception of having experienced adverse drug reactions. This perception of medication side effects may affect their adherence. Finally, this study did not assess patients' health literacy. Patients require sufficient literacy to comprehend medication instructions and calculate accurate medication doses to be taken for the suitable duration. ${ }^{66,67}$ Poor health literacy is linked to a range of unfavorable health outcomes possibly due to improper self-care, incomplete health responsibility, and under-utilization of available healthcare resources. Methods to augment health literacy, rather than providing disease information might be valuable in enhancing medication adherence. 


\section{Conclusion}

Patient education significantly improves adherence. However, in this study, there were no differences between single education intervention and multi-component education intervention in improving medication adherence. Provision of disease information pamphlet with or without directed counseling can equally improve medication adherence of patients with RA.

\section{Data sharing statement}

Individual participant data that underlie the results reported in this article, after deidentification, information pamphlet, and study protocol, are available on request from the corresponding author after article publication.

\section{Acknowledgment}

This study was supported by a research grant from the Thai Rheumatism Association. This manuscript was verified to be correct English by Stephen John Pinder, a native speaker experienced in medical English.

\section{Disclosure}

The authors report no conflicts of interest in this work.

\section{References}

1. Harris ED. Rheumatoid arthritis. Pathophysiology and implications for therapy. N Engl J Med. 1990;322(18):1277-1289.

2. Erickson AR, Cannella AC, Mikuls TR. Clinical Features of Rheumatoid Arthritis. In: Firestein GS, Budd RC, Gabriel SE, McInnes IB, O'Dell JR, editors. Kelley and Firestein's Textbook of Rheumatology. 10 ed. Philadelphia: Elservier, Inc.; 2016:1167-1168.

3. Chaiamnuay P, Darmawan J, Muirden KD, Assawatanabodee P. Epidemiology of rheumatic disease in rural Thailand: a WHO-ILAR COPCORD study. Community oriented programme for the control of rheumatic disease. J Rheumatol. 1998;25(7):1382-1387.

4. Louthrenoo W. An insight into rheumatology in Thailand. Nat Rev Rheumatol. 2015;11(1):55-61.

5. Osiri M, Maetzel A, Tugwell P. The economic burden of rheumatoid arthritis in a developing nation: results from a one-year prospective cohort study in Thailand. $J$ Rheumatol. 2007;34(1):57-63

6. Xia Y, Yin R, Fu T, et al. Treatment adherence to disease-modifying antirheumatic drugs in Chinese patients with rheumatoid arthritis. Patient Prefer Adherence. 2016;10:735-742.

7. Prudente LR, Diniz JS, Ferreira TX, et al. Medication adherence in patients in treatment for rheumatoid arthritis and systemic lupus erythematosus in a university hospital in Brazil. Patient Prefer Adherence. 2016; 10:863-870.

8. Kumar K, Raza K, Nightingale P, et al. Determinants of adherence to disease modifying anti-rheumatic drugs in White British and South Asian patients with rheumatoid arthritis: a cross sectional study. $B M C$ Musculoskelet Disord. 2015;16(1):396.

9. van den Bemt BJ, den Broeder AA, van den Hoogen FH, et al. Making the rheumatologist aware of patients' non-adherence does not improve medication adherence in patients with rheumatoid arthritis. Scand $J$ Rheumatol. 2011;40(3):192-196.

10. Scheiman-Elazary A, Duan L, Shourt C, et al. The rate of adherence to antiarthritis medications and associated factors among patients with rheumatoid arthritis: a systematic literature review and metaanalysis. J Rheumatol. 2016;43(3):512-523.
11. Wong PK. Medication adherence in patients with rheumatoid arthritis: why do patients not take what we prescribe? Rheumatol Int. 2016; 36(11):1535-1542.

12. De Vera MA, Mailman J, Galo JS. Economics of non-adherence to biologic therapies in rheumatoid arthritis. Curr Rheumatol Rep. 2014; 16(11):460.

13. Hovstadius B, Petersson G. Non-adherence to drug therapy and drug acquisition costs in a national population - a patient-based register study. BMC Health Serv Res. 2011;11(1):326.

14. Pascual-Ramos V, Contreras-Yáñez I, Villa AR, Cabiedes J, RullGabayet M. Medication persistence over 2 years of follow-up in a cohort of early rheumatoid arthritis patients: associated factors and relationship with disease activity and with disability. Arthritis Res Ther. 2009; 11(1):R26

15. Brandstetter S, Riedelbeck G, Steinmann M, Loss J, Ehrenstein B, Apfelbacher C. Depression moderates the associations between beliefs about medicines and medication adherence in patients with rheumatoid arthritis: Cross-sectional study. J Health Psychol. 2018;23(9):1185-1195.

16. Harnett J, Wiederkehr D, Gerber R, Gruben D, Bourret J, Koenig A. Primary nonadherence, associated clinical outcomes, and health care resource use among patients with rheumatoid arthritis prescribed treatment with injectable biologic disease-modifying antirheumatic drugs. J Manag Care Spec Pharm. 2016;22(3):209-218.

17. Harrold LR, Briesacher BA, Peterson DAN, et al. Cost-related medication nonadherence in older patients with rheumatoid arthritis. $J$ Rheumatol. 2013;40(2):137-143.

18. Joplin S, van der Zwan R, Joshua F, Wong PK. Medication adherence in patients with rheumatoid arthritis: the effect of patient education, health literacy, and musculoskeletal ultrasound. BioMed Res Int. 2015; 2015:150658

19. Li L, Cui Y, Yin R, et al. Medication adherence has an impact on disease activity in rheumatoid arthritis: a systematic review and meta-analysis. Patient Prefer Adherence. 2017;11:1343-1356.

20. Morgan C, McBeth J, Cordingley L, et al. The influence of behavioural and psychological factors on medication adherence over time in rheumatoid arthritis patients: a study in the biologics era. Rheumatology. 2015;54(10):1780-1791.

21. Rauscher V, Englbrecht M, van der Heijde D, Schett G, Hueber AJ. High degree of nonadherence to disease-modifying antirheumatic drugs in patients with rheumatoid arthritis. $J$ Rheumatol. 2015;42(3): 386-390.

22. Salt E, Frazier SK. Predictors of medication adherence in patients with rheumatoid arthritis. Drug Dev Res. 2011;72(8):756-763.

23. Salt E, Hall L, Peden AR, Home R. Psychometric properties of three medication adherence scales in patients with rheumatoid arthritis. J Nurs Meas. 2012;20(1):59-72.

24. Santoleri F, Sorice P, Lasala R, Rizzo RC, Costantini A. Medication adherence and persistence in the treatment of rheumatoid arthritis with adalimumab and etanercept. Six years of analysis. J Med Econ. 2014; 17(5):320-325.

25. Shetty R, Reddy K, Inam S, Khera K. Impact of medication adherence by using Indian version compliance questionnaire rheumatology (Cqr) and medication adherence report scale (Mars) tools on quality of life of patients with rheumatoid arthritis. Value Health. 2014;17(7):A385.

26. Spruill TM, Ogedegbe G, Harrold LR, et al. Association of medication beliefs and self-efficacy with adherence in urban Hispanic and African-American rheumatoid arthritis patients. Ann Rheum Dis. 2014; 73(1):317-318.

27. Suh YS, Cheon YH, Kim HO, et al. Medication nonadherence in Korean patients with rheumatoid arthritis: the importance of belief about medication and illness perception. The Korean J Intern Med. 2018; 33(1):203-210.

28. Treharne GJ, Lyons AC, Hale ED, Douglas KM, Kitas GD. Predictors of medication adherence in people with rheumatoid arthritis: studies are necessary but non-validated measures of medication adherence are of concern. Rheumatology (Oxford). 2005;44(10):1330. 
29. Tuncay R, Eksioglu E, Cakir B, Gurcay E, Cakci A. Factors affecting drug treatment compliance in patients with rheumatoid arthritis. Rheumatol Int. 2007;27(8):743-746.

30. van den Bemt BJ, van den Hoogen FH, Benraad B, Hekster YA, van Riel PL, van Lankveld W. Adherence rates and associations with nonadherence in patients with rheumatoid arthritis using disease modifying antirheumatic drugs. J Rheumatol. 2009;36(10):2164-2170.

31. van den Bemt BJ, Zwikker HE, van den Ende CH. Medication adherence in patients with rheumatoid arthritis: a critical appraisal of the existing literature. Expert Rev Clin Immunol. 2012;8(4):337-351.

32. Viller F, Guillemin F, Briancon S, Moum T, Suurmeijer T, van den Heuvel W. Compliance to drug treatment of patients with rheumatoid arthritis: a 3 year longitudinal study. J Rheumatol. 1999;26(10): 2114-2122.

33. Zwikker HE, van Dulmen S, den Broeder AA, van den Bemt BJ, van den Ende $\mathrm{CH}$. Perceived need to take medication is associated with medication non-adherence in patients with rheumatoid arthritis. Patient Prefer Adherence. 2014;8:1635-1645.

34. Bruera S, Barbo AG, Lopez-Olivo MA. Use of medication reminders in patients with rheumatoid arthritis. Rheumatol Int. 2016;36(11): $1543-1548$

35. Barton JL, Trupin L, Schillinger D, et al. Use of low-literacy decision aid to enhance knowledge and reduce decisional conflict among a diverse population of adults with rheumatoid arthritis: results of a pilot study. Arthritis Care Res. 2016;68(7):889-898.

36. Depont F, Berenbaum F, Filippi J, et al. Interventions to improve adherence in patients with immune-mediated inflammatory disorders: a systematic review. PLoS One. 2015;10(12):e0145076.

37. Hill J, Bird H, Johnson S. Effect of patient education on adherence to drug treatment for rheumatoid arthritis: a randomised controlled trial. Ann Rheum Dis. 2001;60(9):869-875.

38. Evers AW, Kraaimaat FW, van Riel PL, de Jong AJ. Tailored cognitivebehavioral therapy in early rheumatoid arthritis for patients at risk: a randomized controlled trial. Pain. 2002;100(1-2):141-153.

39. El Miedany Y, El Gaafary M, Palmer D. Assessment of the utility of visual feedback in the treatment of early rheumatoid arthritis patients: a pilot study. Rheumatol Int. 2012;32(10):3061-3068.

40. El Miedany Y, El Gaafary M, El Arousy N, Ahmed I, Youssef S, Palmer D. Arthritis education: the integration of patient-reported outcome measures and patient self-management. Clin Exp Rheumatol. 2012;30(6):899-904.

41. Homer D, Nightingale P, Jobanputra P. Providing patients with information about disease-modifying anti-rheumatic drugs: individually or in groups? A pilot randomized controlled trial comparing adherence and satisfaction. Musculoskeletal Care. 2009;7(2):78-92.

42. Brus HL, van de Laar MA, Taal E, Rasker JJ, Wiegman O. Effects of patient education on compliance with basic treatment regimens and health in recent onset active rheumatoid arthritis. Ann Rheum Dis. 1998;57(3):146-151.

43. Greenley RN, Kunz JH, Walter J, Hommel KA. Practical strategies for enhancing adherence to treatment regimen in inflammatory bowel disease. Inflamm Bowel Dis. 2013;19(7):1534-1545.

44. Roter DL, Hall JA, Merisca R, Nordstrom B, Cretin D, Svarstad B. Effectiveness of interventions to improve patient compliance: a metaanalysis. Med Care. 1998;36(8):1138-1161.

45. National Collaborating Centre for Primary Care. Medicines Adherence: Involving Patients in Decisions About Prescribed Medicines and Supporting Adherence; 2009. Available from: https://www.nice.org.uk/ guidance/cg76. Accessed October 25, 2018

46. Sakthong P, Sonsa-Ardjit N, Sukarnjanaset P, Munpan W, Suksanga P. Development and psychometric testing of the medication taking behavior tool in Thai patients. Int J Clin Pharm. 2016;38(2):438-445.

47. Herdman M, Gudex C, Lloyd A, et al. Development and preliminary testing of the new five-level version of EQ-5D (EQ-5D-5L). Qual Life Res. 2011;20(10):1727-1736.
48. Prevoo MLL, van't Hof MA, Kuper HH, van Leeuwen MA, van de Putte LBA, van Riel PLCM. Modified disease activity scores that include twenty-eight-joint counts development and validation in a prospective longitudinal study of patients with rheumatoid arthritis. Arthritis Rheum. 1995;38(1):44-48.

49. Nilchaikovit T, Lotrakul M, Phisansuthideth U. Development of Thai version of hospital anxiety and depression scale in cancer patients. J Psychiatr Assoc Thailand. 1996;41(1):18-30.

50. Zigmond AS, Snaith RP. The hospital anxiety and depression scale. Acta Psychiatr Scand. 1983;67(6):361-370.

51. Broadbent E, Petrie KJ, Main J, Weinman J. The brief illness perception questionnaire. J Psychosom Res. 2006;60(6):631-637.

52. Sowattanangoon N. Brief Illness Perception Scale (Thai version); 2008. Available from: http://www.uib.no/ipq/html/b-ipq.html. Accessed October 25, 2018.

53. Tangwongchai S, Phanasathit M, Charernboon T, et al. The validity of Thai version of the Montreal Cognitive Assessment (MoCA-T). Dement Neuropsychol. 2009;3(2):172. Available from: http://demneuropsy.com.br/imageBank/pdf/dnv03n02a11.pdf. Accessed January 3, 2019.

54. Train The Brain Forum Committee. Thai Mental State Examination (TMSE). Siriraj Med J. 1993;45(6):1695-1703.

55. Ngamjarus C. n4Studies: Sample Size Calculation for an Epidemiological Study on a Smart Device. Siriraj Med J. 2016;68(3):160-170.

56. Moshkovska T, Stone MA, Smith RM, Bankart J, Baker R, Mayberry JF. Impact of a tailored patient preference intervention in adherence to 5-aminosalicylic acid medication in ulcerative colitis: results from an exploratory randomized controlled trial. Inflamm Bowel Dis. 2011;17(9): 1874-1881.

57. Cook PF, Emiliozzi S, El-Hajj D, McCabe MM. Telephone nurse counseling for medication adherence in ulcerative colitis: a preliminary study. Patient Educ Couns. 2010;81(2):182-186.

58. Elkjaer M, Shuhaibar M, Burisch J, et al. E-health empowers patients with ulcerative colitis: a randomised controlled trial of the web-guided 'constant-care' approach. Gut. 2010;59(12):1652-1661.

59. Moss AC, Chaudhary N, Tukey M, et al. Impact of a patient-support program on mesalamine adherence in patients with ulcerative colitis - a prospective study. J Crohns Colitis. 2010;4(2):171-175.

60. Zwikker HE, van den Ende CH, van Lankveld WG, et al. Effectiveness of a group-based intervention to change medication beliefs and improve medication adherence in patients with rheumatoid arthritis: a randomized controlled trial. Patient Educ Couns. 2014;94(3):356-361.

61. Treharne GJ, Lyons AC, Hale ED, Douglas KM, Kitas GD. 'Compliance' is futile but is 'concordance' between rheumatology patients and health professionals attainable? Rheumatology. 2006;45(1):1-5.

62. Peveler R, George C, Kinmonth AL, Campbell M, Thompson C. Effect of antidepressant drug counselling and information leaflets on adherence to drug treatment in primary care: randomised controlled trial. $B M J$. 1999;319(7210):612-615.

63. Helliwell PS, O'Hara M, Holdsworth J, Hesselden A, King T, Evans P. A 12-month randomized controlled trial of patient education on radiographic changes and quality of life in early rheumatoid arthritis. Rheumatology. 1999;38(4):303-308.

64. Dunbar J, Dunning EJ, Dwyer K. Compliance measurement with arthritis regimen. Arthritis Care Res. 1989;2(3):A8-A16.

65. Pullar T. Compliance with drug therapy. Br J Clin Pharmacol. 1991; 32(5):535-539.

66. Miles S, Davis T. Patients who can't read. Implications for the health care system. JAMA. 1995;274(21):1719-1720.

67. Health literacy: report of the Council on Scientific Affairs. Ad Hoc Committee on Health Literacy for the Council on Scientific Affairs, American Medical Association. JAMA. 1999;281(6):552-557. 
Patient Preference and Adherence

Dovepress

\section{Publish your work in this journal}

Patient Preference and Adherence is an international, peer-reviewed, open access journal that focuses on the growing importance of patient preference and adherence throughout the therapeutic continuum. Patient satisfaction, acceptability, quality of life, compliance, persistence and their role in developing new therapeutic modalities and compounds to optimize

Submit your manuscript here: http://www.dovepress.com/patient-preference-and-acherence-journa clinical outcomes for existing disease states are major areas of interest for the journal. This journal has been accepted for indexing on PubMed Central. The manuscript management system is completely online and includes a very quick and fair peer-review system, which is all easy to use. Visit http://www. dovepress.com/testimonials.php to read real quotes from published authors. 